\title{
Goal-oriented agents in a market ${ }^{\text {is }}$
}

\author{
Inés Macho-Stadler ${ }^{a}$, David Pérez-Castrillo ${ }^{\text {a, }}$, Nicolas Quérou ${ }^{\text {b }}$ \\ ${ }^{\text {a }}$ Univ. Autonoma de Barcelona and Barcelona GSE, Barcelona, Spain \\ ${ }^{\mathrm{b}}$ CEE-M, Univ. Montpellier, CNRS, INRAe, Institut Agro, Montpellier, France
}

\section{A R T I C L E I N F O}

JEL Classification numbers:

D82

D86

Keywords:

Goal-oriented agents

Incentives

Matching market

\begin{abstract}
A B S T R A C T
We consider a market where "standard" risk-neutral agents coexist with "goal-oriented" agents who, in addition to the expected income, seek a high-enough monetary payoff (the "trigger") to fulfill a goal. We analyze a two-sided one-to-one matching model where the matching between principals and agents and incentive contracts are endogenous. In any equilibrium contract, goaloriented agents are matched with the principals with best projects and receive the trigger with positive probability. Moreover, goal and monetary incentives are complementary: goal-oriented agents receive stronger monetary incentives. Finally, we discuss policy interventions in relevant environments.
\end{abstract}

\section{Introduction}

Individuals sometimes need a certain amount of money to achieve a goal. For instance, a person with a plan to start a promising business is often required to cover a percentage of the initial investment because no financier would lend him or her the total amount needed. Thus, this person needs a minimum capital level to implement the idea and become an entrepreneur. Similarly, an individual may be willing to achieve high-school or college education, or may be willing that a descendant achieves such goal. However, education requires paying expensive tuition fees up front, and it also implies wage losses during schooling years. Hence, even though education may be an excellent investment opportunity for this person for both monetary and personal reasons, he or she first needs to accumulate some savings because the option to borrow for college fees is not available in many countries, and it may be quite limited in many others. Finally, other relevant examples rely on purely behavioral motives. This is the case, for example, when an agent's goal is to reach an aspiration-based reference compensation level.

In this paper, we study the implications of the presence of such "goal-oriented" workers in a market. In an environment where goaloriented agents coexist with standard ones, we analyze how the heterogeneity in the agents' population affects the equilibrium matching and contracts. We also show that the presence of goal-oriented agents creates a wedge between different markets, which results in potentially important feedback effects of policy interventions.

More precisely, we consider a labor market composed by a population of risk-neutral principals and a population of agents who are subject to limited liability. The agents' population include both "standard" risk-neutral agents who maximize expected income and goal-oriented agents who, in addition to the expected income, care about the possibility of obtaining a high-enough monetary payoff to

We thank Joaquín Gómez-Miñambres and two reviewers for their very helpful comments. We acknowledge financial support from the GREEN-Econ research project (grant ANR-16-CE03-0005), Spanish Ministry of Science and Innovation and Feder (PGC2018-094348-B-I00 and Severo Ochoa program CEX2019-000915-S), Generalitat de Catalunya (2017SGR-711), and ICREA under the ICREA Academia programme.

* Corresponding author.

E-mail addresses: ines.macho@uab.es (I. Macho-Stadler), david.perez@uab.es (D. Pérez-Castrillo), nicolas.querou@umontpellier.fr (N. Quérou). 
be able to fulfill their goal. That is, such a monetary payoff constitutes the "trigger" of the goal. Each principal in the market can enter into a partnership with an agent in order to develop the principal's project, which ends up being either a success or a failure, the probability of a successful outcome depending on the agent's effort. However, any principal-agent relationship is subject to moral hazard because the agent's effort is not verifiable. Hence, the two partners sign a contract including a payment that can be contingent on the outcome. An important feature of our model is that the goal of the goal-oriented agents is external to the market we study here: a principal who hires a goal-oriented agent cannot influence the characteristics of this goal, although she can influence the possibility that the agent reaches the trigger through the monetary compensation offered in the contract.

Thus, we analyze a two-sided one-to-one matching model where the matching (who partners with whom) as well as the incentive contracts implemented in the formed partnerships are endogenous. ${ }^{1}$ In this market, we look for the equilibrium, or stable outcomes. ${ }^{2}$ One important property of equilibrium contracts is that they are Pareto optimal.

Goal-oriented agents have stronger incentives to work hard than standard agents if the contract allows for the possibility to reach the trigger. Thus, goal-oriented agents may be more attractive to principals than standard agents. We assume an environment where the agents' population is larger than that of the principals, but where the number of goal-oriented agents is low. In this environment, all goal-oriented agents will be matched at the equilibrium.

We show that, in any equilibrium contract signed in a partnership involving a goal-oriented agent, the worker receives the trigger value with a positive probability. If the trigger value is not too high, then the equilibrium contract includes, in addition to the base payment, a bonus that is always paid in case of success and ensures that, following a successful outcome, the compensation level is higher than the trigger value. On the other hand, if the trigger value is high, then the bonus (in case of success) is not always paid. The equilibrium contract specifies a bonus such that the agent receives the trigger value in case of success, but the bonus is only paid with some probability. This contract structure improves on the agent's incentives and expected utility with respect to another contract specifying a lower bonus that would always be paid in case of success.

At the equilibrium, goal-oriented agents work harder than standard agents for two reasons. First, as we have seen, the equilibrium contract always opens up the possibility that the goal be achieved in case of success which, ceteris paribus, induces a goal-oriented agent to exert a higher effort than a standard agent. Second, and more interestingly, at the equilibrium outcome, goal and monetary incentives are complementary: goal-oriented agents receive stronger monetary incentives than standard agents. Indeed, the competition among principals to hire the goal-oriented agents forces them to offer to those agents a higher expected utility than what the standard agents get. In the equilibrium contract, the increase in utility translates into higher incentives. Since goals are exogenous, we here stress a causality effect from goals to incentives.

We also show that the more profitable a principal's project is, the more she benefits from hiring a goal-oriented agent instead of a standard one. As a result, at the equilibrium, the goal-oriented agents work for the most profitable projects. That is, the matching between principals and agents is positive assortative.

Finally, we use our comparative statics results to discuss the potential effects of some policy interventions. We provide this discussion in three relevant examples: when the agent's goal is to become an entrepreneur, when his goal is to buy an asset with persistent benefits, and when the goal is due to an aspiration-based reference compensation level.

Consider, for instance, the case where some agents' goal is to become entrepreneurs. When working in the market analyzed here, a goal-oriented agent's extra utility would correspond to the sum of the expected profit that the agent obtains if he could start the venture and of non-monetary benefits associated to being an entrepreneur. We first show that a policy that would increase the base salary in our market would lead to a higher effort level exerted by goal-oriented agents, but also to an increase in the probability that these agents become entrepreneurs. A second policy that would provide a subsidy to new entrepreneurs, or that would decrease the total investment needed to carry out the agent's project idea, would increase the goal-oriented agents' motivation to exert effort in their current job, which in turn would positively impact principals' profit. Moreover, it would also increase these agents' utility, as they would be more likely to become entrepreneurs. Yet, such a subsidy scheme would need to be funded by the government, and the positive effect on the goal-oriented agents' utility would need to be traded off with the cost resulting from the use of public funding. A final option would be to affect the taxes and fees paid by entrepreneurs (or self-employed people), affecting the expected income of these activities. Reducing the charges to be paid by entrepreneurs would increase the number of entrepreneurs and have an indirect positive effect on the market in which these individuals were employed as agents.

Thus, there are externalities from a policy implemented in the present labor market on the entrepreneurship activity, and policies related to the entrepreneurship activity have in turn external effects on the present labor market.

The rest of the paper is organized as follows. Section 2 discusses the related literature. Section 3 presents the model and the equilibrium concept. Section 4 provides the results. In Section 5, we discuss the impact of some policy interventions. Finally, Section 6 concludes. All the proofs are provided in an Appendix.

\footnotetext{
${ }^{1}$ See the seminal papers by Gale and Shapley (1962) and Shapley and Shubik (1971). The first paper introduces the marriage and college admission models (two-sided one-to-one and many-to-one matching models without transfers) whereas the second paper introduces the assignment game (two-sided one-to-one matching models with perfectly transferable utility).

${ }^{2}$ In our framework, utility is partially transferable among participants. Demange and Gale (1985) study the stable outcomes in this non-linear environment.
} 


\section{Related literature}

Our model combines three features: a moral hazard problem with limited liability, a market where agents and principals match and sign contracts, and agents exhibiting an additional motivation.

Limited liability has been extensively used to study moral hazard problems. Given its tractability, considering that agents are risk neutral and protected by limited liability (see, e.g., Innes, 1990; Poblete \& Spulber, 2012) allows to introduce moral hazard problems in complex models. This approach has been particularly helpful to analyze principal-agent relationships in developing countries, where agents' limited wealth is an important concern, or in markets in which it is critical to have some initial capital level. For example, Shetty (1988) considers the market between tenants and landlords, and analyzes the influence of tenants' wealth on tenancy contracts. Quérou, Soubeyran, and Soubeyran (2020) study a situation where the agent's effort implies not only disutility but also monetary costs, in such a way that limited resources restrict the agent's capability to exert effort.

The second feature of our analysis is to introduce a moral hazard problem in a market where principals and agents meet. Several papers have considered principal-agent matching markets under moral hazard. ${ }^{3}$ For example, Besley and Ghatak (2005) consider mission-oriented and profit-oriented principals together with agents who may or may not support the mission. Chakraborty and Citanna (2005) study an environment where individuals differ in their wealth endowment and they can either remain self-employed or engage in productive matches with another individual. Dam and Pérez-Castrillo (2006) analyze the influence of the distribution of the agents' wealth on the efficiency of the matching formed. As in those papers, we assume that information about the characteristics of the principals and the agents is symmetric. One feature of our matching model is that utility is not perfectly transferable. For these environments, Legros and Newman (2007) provide results highlighting whether the matching is positive or negative assortative depending on the characteristics of the participants on both sides of the market. By contrast, our main focus in this paper lies in understanding the characteristics of the contracts signed by the parties.

The third feature of our model is the interplay between monetary rewards and the existence of an additional motivation resulting from a personal goal. In our model, a sizable monetary bonus triggers an extra utility for some agents, the goal-oriented ones, because it allows these agents to fulfill their goal. This view is complementary to the one described in a large literature studying how monetary payments may reduce the agents' "intrinsic" motivation when they care about what they do, in addition to how much they are paid (see e.g., Deci \& Ryan, 1985 \& Kreps, 1997). ${ }^{4}$ In that literature, monetary incentives crowd out the agent's intrinsic motivation. By contrast, in our setup the monetary bonus may enable to fulfill the goal: hence, monetary incentives may trigger the incentives derived from the goal.

The idea that agents may have goals, or reference points, that affect their behavior has been studied in several papers. For example, Köszegi and Rabin (2006) consider that employees may have reference-dependent preferences, where the reference point corresponds to the agents' expectation about outcomes. Gómez-Miñambres (2012), Corgnet, Gómez-Miñambres, and Hernán-Gonzalez (2015), and Brookins, Goerg, and Kube (2017) study isolated principal-agent environments where a goal (in terms of output obtained by the agent) can be set either by the principal (in the first two contributions) or by the agent himself (in the third contribution). The agent derives an intrinsic utility when fulfilling the goal and may derive an intrinsic disutility if he does not achieve the goal. In contrast, the agent's goal in our setup is exogenous, idiosyncratic, and not related to the outcome of the relationship but to the wage. Moreover, we study equilibrium contracts where goal-oriented agents coexist with standard agents. Corgnet et al. (2015) and Brookins et al. (2017) find, as we do, a complementarity between the strength of the incentives and the magnitude of the agents' goal. In these contributions, an increase in the exogenous strength of incentives leads (either the principal or the agent) to select a higher goal level. In our paper, the causality is reversed: an increase in the exogenous magnitude of the goal (which is unrelated to the output) yields stronger incentives in the goal-oriented agent's contract. Moreover, this complementarity only occurs at the market equilibrium: it would not hold in an isolated principal-agent relationship. ${ }^{5}$

To our knowledge, the closest contribution to ours is Ghatak, Morelli, and Sjostrom (2001). They consider a market where agents experience an extra motivation from the possibility to become principals in the future. More precisely, Ghatak et al. (2001) study an overlapping generations model of a principal-agent problem in which contracts are characterized using a general equilibrium approach. In their model, all young workers are identical but, depending on their performance, they have different investment possibilities when being old. Old agents can choose between becoming principals (at a cost) or remaining workers. Imperfections in the credit market yield rents to principals (entrepreneurs), and these rents induce an extra motivation for all young workers. They show that the potential option to become a principal in the market induces higher effort levels and increases welfare. The authors stress the fact that policies reducing market credit imperfections (or redistributing income) may reduce welfare by dampening this effect.

We model the extra motivation in a similar way as Ghatak et al. (2001) do. In contrast to them, first, agents are ex-ante asymmetric in our model; not all agents in the market exhibit the motivation to become entrepreneurs. Second, we consider a static model where the agents may achieve their goal in a different market than the one they work in. This allows to analyze other interesting scenarios besides entrepreneurship and to study the external effects induced by policies in one market over the other market. Third, we solve for

\footnotetext{
3 For a recent review, see Macho-Stadler and Pérez-Castrillo (in press).

${ }^{4}$ For more details see Köszegi (2014) and Macho-Stadler and Pérez-Castrillo (2018), as well the references included in these survey papers.

5 Several contributions have focused on loss-averse agents (see for instance De Meza \& Webb, 2007; Dittmann, Maug, \& Spalt, 2010; Herweg, Muller, \& Weinschenk, 2010). In contrast to the literature discussed above, where achieving the goal yields an extra utility to the agents (an intrinsic motivation), a loss-averse agent experiences disutility when he does not achieve the goal. We discuss the consequences of the existence of a form of loss aversion in our environment in subsection 4.3 .
} 
a larger class of contracts. We consider random contracts, including a combination of a base salary and a bonus payment that the agent receives only with some probability in case of success. ${ }^{6}$ Finally, assuming that only some agents are goal-oriented allows to discuss how the matching and the contracts depend on the scarcity or abundance of this type of agents in the economy.

\section{The setting}

\subsection{The model}

We consider a labor market with $n$ risk-neutral principals and $m$ agents. In this market, each principal and each agent can form a partnership, that is, they can be matched. Each of these players can only take part in one partnership. Alternatively, any principal or agent can remain unmatched.

If a principal and an agent take part in a relationship, they develop a project. The outcome of the project $x$ can be either a success or a failure. If successful, the relationship yields a return $R>0$, while failure results in zero return. ${ }^{7}$ The agent's effort $e, e \in[0,1]$, determines the probability of success $p(e)=e$ of the relationship. The cost of effort $e$ for the agent is $\frac{v}{2} e^{2}$. The effort is not verifiable. The principal is the residual claimant of the output and designs the contract to address this moral hazard problem.

The contract exhibits three features: a base salary $w$ to be paid by the principal to the agent, a bonus $\Delta \geqslant 0$ that the agent may get in case of success, and the probability $p \in[0,1]$ with which the agent gets the bonus in case of success.

The main idea to address the classical moral hazard model is to provide the agent with monetary incentives through a contractual scheme $(w, \Delta, p) .^{8}$ In our paper, we consider that some agents may also have some additional motivation related to the wage they receive. We assume that obtaining a certain amount of money allows some agents to achieve a goal, for instance: becoming an entrepreneur (in another market), buying an asset with persistent benefits, or fulfilling some aspiration. For any such reasons, an agent may obtain an extra utility (his extra motivation) if his payoff lies above a certain threshold level, which we denote by $z, z>0$. That is, $z$ is the trigger of the extra motivation. We denote by $k$ the extra utility that such an agent gets if he is able to fulfill his goal, that is, if he receives a payment that is at least equal to $z$.

Thus, in the present market an agent may be risk neutral and thus he may not have a personal goal: We will refer to such an agent as a standard agent. Or an agent may have a personal goal, which is described by the parameters $(z, k)$. In this case, his utility function is linear in money, except at the trigger value $z$, where there is a jump upwards: We will refer to such an agent as a goal-oriented agent. Hence, a standard agent corresponds to an agent with extra utility equal to 0 . Formally, the utility of a standard $(S)$ and that of a goaloriented $(G)$ agent who sign a contract $(w, \Delta, p)$ and exert effort level $e$ are, respectively:

$$
\begin{aligned}
& u^{S}(w, \Delta, p ; e)=w+p e \Delta-\frac{v}{2} e^{2}, \\
& u^{G}(w, \Delta, p ; e)=\left(w+I_{w} k\right)+p e\left(\Delta+I_{w+\Delta} k\right)-\frac{v}{2} e^{2},
\end{aligned}
$$

where $I_{w}=0$ if $w<z ; I_{w}=1$ if $w \geqslant z ; I_{w+\Delta}=0$ if either $w+\Delta<z$ or $w \geqslant z$; and $I_{w+\Delta}=1$ if $w<z \leqslant w+\Delta$.

The number of goal-oriented agents is $m^{G}$ and that of standard agents is $m^{S}$, with $m^{G}+m^{S}=m$. We assume that $m>n>m^{G}$, that is, the number of principals is smaller than that of the agents, but there are less goal-oriented agents than there are principals in the present market. Information about the agents' type is symmetric.

The principal's expected profit under the contract $(w, \Delta, p)$ when the agent exerts effort $e$ is:

$$
\Pi(w, \Delta, p ; e)=e R-p e \Delta-w .
$$

The expression of the principal's profit does not depend directly on the agent's characteristics, although it may depend indirectly on these characteristics through the agent's choice of effort level.

We assume that agents are protected by limited liability so that their final payoff cannot be lower than a minimum wage, which we denote $\underline{w}$. Then, a feasible contract must satisfy the limited liability constraints (LLC):

$$
w \geqslant \underline{w} \text { and } w+\Delta \geqslant \underline{w} \text {. }
$$

The contract must be designed by taking into account that the agent will choose the level of exerted effort. He supplies the effort level $e$ that maximizes his expected utility, given the contract $(w, \Delta, p)$. The incentive compatibility constraint (ICC) for a standard agent is:

\footnotetext{
${ }^{6}$ Payments that are not only tied to outcomes but also to a random device that does not depend on the agent's effort are usually not considered because they are either dominated by output-contingent contracts, if the agents are risk averse, or equivalent, if the agents are risk neutral. This property does not hold for goal-oriented agents, whether they are risk neutral or risk averse, if it allows fulfilling the goal with some probability.

7 We here consider the case where the technology is homogeneous. We will extend the analysis to the case of heterogeneous technologies in Section 4.2.

${ }^{8}$ In order for a random scheme to be verifiable, the principal must be able to commit to the probability with which she will pay the bonus. She can delegate to a third party the rights to run a lottery or she can refer to the outcome of a third-party lottery.
} 


$$
e^{s}(w, \Delta, p)=\frac{p \Delta}{v}
$$

For a goal-oriented agent, the ICC depends on the payments $w$ and $w+\Delta$ and on the trigger value $z$ :

$$
e^{G}(w, \Delta, p)=\left\{\begin{array}{l}
\frac{p(\Delta+k)}{v} \text { if } w<z \leqslant w+\Delta \\
\frac{p \Delta}{v} \text { if either } w+\Delta<z \text { or } z \leqslant w .
\end{array}\right.
$$

Indeed, if $w<z \leqslant w+\Delta$ holds then a goal-oriented agent obtains the extra utility $k$ in case of success, which provides him with extra incentives that add up to those provided by the bonus $\Delta$.

Assumption 1 specifies some restrictions on the parameter values:

Assumption 1. (i) $\underline{w}<z$; (ii) $\underline{w}<\frac{1}{8 v} R^{2}$; and (iii) $k+z-\underline{w}<v$ and $R \leqslant 2(v-\sqrt{v k})$.

Part (i) ensures that the base salary is not sufficient to trigger the extra utility, whereas (ii) is a necessary condition to ensure that the principal derives positive profits when she signs a contract with a standard agent. Condition (iii) ensures that the effort level specified in the equilibrium contract lies in the interval $[0,1]$.

Finally, if an agent is not involved in any partnership, then the utility that he obtains outside the market (or when he is not matched) is $U^{o}=0$. Similarly, a principal obtains zero profit if she is not matched to an agent, that is, $\Pi^{o}=0$.

\subsection{The equilibrium concept}

In our market, any principal can contract with any agent, and any agent can partner with any principal. Therefore, the (two-sided one-to-one) matching between principals and agents is endogenous. The endogeneity of the matching process implies that both principals' profits and agents' utility levels are determined by market competition. Moreover, the contract signed within any partnership is also endogenous. Thus, an outcome consists of a set of partnerships, a set of unmatched principals and agents, and a contract for each partnership.

The equilibrium concept that we use is the classic concept of stability (see Shapley \& Shubik, 1971, for the case of transferable utility and Demange \& Gale, 1985, for the non-linear case). An outcome is an equilibrium outcome if (i) each partner within a relationship obtains at least the same profit/utility level as when she/he remains unmatched and (ii) there is no principal-agent pair and no contract such that both the principal and the agent are better-off under the new contract than under the equilibrium outcome.

Contracts that correspond to an equilibrium outcome satisfy a property that will be useful in this analysis: any such contract must be Pareto optimal. Otherwise, the two members of the partnership considered could deviate and sign a mutually beneficial contract.

\section{Characterization of equilibrium outcomes}

\subsection{Uniform technology}

In this subsection, we consider that all principals share the same technology (the project returns take on the same value $R$ for all principals). The agents only differ in whether they are standard or goal-oriented (but they are identical within each group).

Goal-oriented agents are more appealing to the principals than standard agents because of the extra utility that they can obtain, and because of the extra incentives that result from the possibility to fulfill the goal. This implies that, at the equilibrium, a goal-oriented agent cannot be unmatched while a standard agent is matched. Moreover, Assumption 1 (ii) ensures that a partnership between a principal and a standard agent is always profitable. Given that we consider a market where $0<m^{G}<n<m$, it is necessarily the case that, at an equilibrium outcome: (a) the $n$ principals are matched (because there is surely an agent who is unmatched and the partnerships are profitable); (b) the $m^{G}$ goal-oriented agents are also matched (because some principal is certainly matched with a standard agent and she could deviate with any unmatched goal-oriented agent); and (c) some standard agents are matched and some others are not (because $m>n>m^{G}$ and the matching is one-to-one).

The previous properties can be used to characterize the equilibrium contract offered to the standard agents who are matched. We know that it must be Pareto optimal. Moreover, the size of standard agents' sub-population is such that the principal can sign the best contract for her that ensures that the agent gets his outside option $U^{o}=0$. The characterization of the optimal contract then follows from standard arguments.

Lemma 1. At the equilibrium outcome, in any partnership between a principal and a standard agent:

(i) the contract is $C^{S^{*}} \equiv\left(w=\underline{w}, \Delta=\frac{1}{2 p} R, p\right)$ with $p \in(0,1]$;

(ii) the principal's profit is $\Pi^{S^{*}} \equiv \Pi^{S}\left(C^{S^{*}}\right)=\frac{1}{4 v} R^{2}-\underline{w}$;

(ii) the agent's effort level is $e^{S}=\frac{R}{2 v}$ and his expected utility is $U^{S^{*}} \equiv U^{S}\left(C^{S^{*}}\right)=\frac{1}{8 v} R^{2}+\underline{w}$. 
When a principal hires a standard agent under moral hazard, and random payments are possible, several contracts are optimal. We consider without loss of generality that the optimal contract is $\left(w^{S}=\underline{w}, \Delta^{S}=\frac{1}{2} R, p^{S}=1\right)$.

Lemma 1 states that standard agents who get an offer obtain the expected utility level $U^{S^{*}}$ in the market. This is higher than their outside utility level $U^{o}$ since, as it is common in situations characterized by limited liability, they obtain a rent. The rationale is that the principals are interested in providing incentives to induce effort supply, and the effect of the limited liability constraint is stronger than that of the participation constraint, which is not binding at the optimal contract.

Although hiring a goal-oriented agent is a priori more profitable for a principal, the competition between the principals implies that those who hire them cannot benefit from the potential extra surplus. Two identical principals must obtain the same equilibrium profit: if principal $P_{1}$ would obtain lower profits than principal $P_{2}$, then she could deviate by offering a slightly better contract to the agent matched with $P_{2}$. As such, if there is any extra surplus, this will translate into a higher utility level for these agents. Lemma 2 summarizes this discussion.

Lemma 2. The principals hiring goal-oriented agents obtain equilibrium profits $\Pi^{G}=\Pi^{S^{*}}=\frac{1}{4 v} R^{2}-\underline{w}$.

We now provide some properties of the contract signed by the goal-oriented agents at the equilibrium. The equilibrium contract $C^{G}=(w, \Delta, p)$ designed for such an agent might satisfy one of the following sets of conditions.

First, the contract might be such that $w+\Delta<z$ is satisfied. In this case, the goal-oriented agent is similar to a standard agent.

Second, the contract might provide extra incentives: the payment scheme might satisfy $w<z \leqslant w+\Delta$. Here, the agent gets incentives to work because $\Delta>0$ and he also has extra incentives to work because he obtains $k>0$ with probability $p$ in case of success. Moreover, because he obtains the extra utility $k$ with some probability, the agent's utility level is higher than the utility level a standard agent would obtain under the same contract.

Finally, the contract might specify a payment higher than $z$ for any outcome: $w \geqslant z$ is satisfied. In this case, the contract does not provide any additional incentive but the goal-oriented agent obtains an extra utility $k$ compared to the utility level that a standard agent would obtain under the contract.

Lemma 3 highlights that the equilibrium contract is designed such that conditions $w<z \leqslant w+\Delta$ are satisfied. It also states the intuitive property that the base salary $w$ is always equal to the minimum salary $\underline{w}$.

Lemma 3. The equilibrium contract for a goal-oriented agent satisfies $w=\underline{w}, p \in(0,1]$, and $\Delta \geqslant z-\underline{w}$.

Lemma 3 has useful implications for the characterization of the equilibrium contract signed in any partnership involving a principal and a goal-oriented agent. Since the contract is Pareto optimal, we can compute it either by maximizing the principal's profit subject to the agent's participation constraint, or by maximizing the agent's expected utility subject to the constraint that the principal obtains a given profit level. We focus on the second program because Lemma 2 characterizes the equilibrium profit level obtained by the principals. Moreover, Lemma 3 ensures that, at the equilibrium contract, a goal-oriented agent can achieve his goal with some probability in case of success since $w=\underline{w}<z$ and $\Delta \geqslant z-\underline{w}$ are satisfied. Therefore, in an equilibrium contract $(\underline{w}, \Delta, p)$, we deduce that $e=\frac{p(\Delta+k)}{v}$ is the agent's effort level.

The equilibrium contracts, principals' profits and agents' utility levels are characterized in Theorem 1 for any partnership. The equilibrium contract offered to the goal-oriented agents can take on two different forms, depending on whether the project value $R$ is higher or lower than a threshold, which we denote $\widehat{R}$ :

$$
\widehat{R} \equiv 2(k+z-\underline{w}-\sqrt{k(k+z-\underline{w})}) .
$$

Theorem 1. Under Assumption 1, at an equilibrium outcome:

(a) Standard and goal-oriented agents sign the following contracts, respectively:

$$
\begin{gathered}
C^{S}= \\
\left(w=\underline{w}, \Delta=\frac{R}{2}, p=1\right) . \\
C^{G}=\left\{\left(w=\underline{w}, \Delta=z-\underline{w}, p=\frac{R}{2(z-\underline{w})}\left(1+\frac{\sqrt{k}}{\sqrt{k+z-\underline{w}}}\right)\right) \text { if } R \leqslant \widehat{R}\left(w=\underline{w}, \Delta=\frac{1}{2}(R+\sqrt{k(2 R+k)}-k), p=1\right) \text { if } R \geqslant \widehat{R} .\right.
\end{gathered}
$$


(b) All principals obtain profit

$$
\Pi=\frac{1}{4 v} R^{2}-\underline{w} .
$$

(c) Standard agents (if they are matched) and goal-oriented agents obtain the following utility levels, respectively:

$$
\begin{gathered}
U^{S}= \\
\frac{1}{8 v} R^{2}+\underline{w} . \\
U^{G}=\left\{\frac{1}{8 v}\left(\frac{R}{z-\underline{w}}(k+z-\underline{w}+\sqrt{k(k+z-\underline{w})})\right)^{2}+\underline{w} \text { if } R \leqslant \widehat{R} \frac{1}{8 v}(R+k+\sqrt{k(2 R+k)})^{2}+\underline{w} \text { if } R \geqslant \widehat{R} .\right.
\end{gathered}
$$

For values of $R$ lower than $\widehat{R}$, the probability of getting the bonus $\Delta=z-\underline{w}$ is smaller than one; for larger values of $R$, the agent gets a bonus larger than $z-\underline{w}$ with probability one. Note that the optimal contract is continuous at $R=\widehat{R}$ : the corresponding expression is $(w=\underline{w}, \Delta=z-\underline{w}, p=1)$. The goal-oriented agent's expected utility $U^{G}$ is also continuous and it is increasing in $R{ }^{9}$

Note also that the cut-off $\widehat{R}$ in Theorem 1 is decreasing in $k$ and increasing in $z$. The larger the extra utility from reaching the goal $k$, the more valuable is the agent to the principal; hence, the larger the set of project values $R$ that correspond to contracts for which the agent's reward is larger than $z$ in case of success and the bonus is always paid in case of success. On the other hand, since the cut-off is increasing in $z$, the use of this type of contract is less prevalent when the trigger value is higher.

We now discuss the characteristics of the equilibrium contracts stated in Theorem 1. First, for sufficiently high values of $R(R \geqslant \widehat{R})$ the optimal contract exhibits an interesting and non straightforward property. The intuition suggests that, since goal-oriented agents are easier to motivate than standard ones, the optimal contract might stipulate a lower bonus for goal-oriented agents. Indeed, if one considers an isolated principal-agent contract, goal and monetary incentives are either strict substitutes (for most cases) or independent (for intermediate project values). Yet, when there are market interactions, it is easily checked that $\Delta>\frac{R}{2}$ if $k>0$ holds. That is, at the equilibrium outcome, goal and monetary incentives are complementary. The reason is that goal-oriented agents are more appealing to the principals than standard agents. Given that the principals compete for contracting with them, the goal-oriented agents obtain a higher equilibrium utility level, which translates into higher incentives rather than into a higher fixed payment, because higher incentives result in an increased surplus.

Second, for low values of $R(R<\widehat{R})$ the principals still benefit from the extra motivation derived from the existence of a goal. However, given that the trigger value $z$ is high relative to $R$, a principal would obtain lower profits than $\Pi^{S^{*}}$ if she would always pay $z$ in case of success. Hence, she offers a contract where she pays $z$ in case of success, but with a probability that is lower than one. Therefore, a random contract is optimal and offered at the equilibrium.

Note that, in our model, there is a sharp discontinuity in the payoff leading to a unique goal. A reasonable concern is whether our result is robust to situations where the goal might be achieved with several intensity levels. A business project may be developed with different levels of initial investment, and education of different quality levels can be achieved in colleges charging different tuition fees. In those situations, the extra utility obtained by the agent depends on the amount of money he can allocate to the goal. The qualitative results that we present also hold in this type of environment. For instance, consider a goal that can be achieved for two intensity levels. If the agent receives an amount of money $z_{1}$ then he obtains an extra utility of $k_{1}$, whereas the extra utility is $k_{2}$ if he receives $z_{2}$, with $z_{2}>z_{1}$ and $k_{2}>k_{1}$. In this environment, the contract between principal and agent may include, in addition to the base salary (which will also be $w=\underline{w}$ ) two bonuses $\Delta_{1}$ and $\Delta_{2}$ to be paid in case of success with probabilities $p_{1}$ and $p_{2}$, with $p_{1}+p_{2} \leqslant 1$. If $R$ is not too large (which would correspond to a condition similar to $R \leqslant \widehat{R}$ in Theorem 1), then by the same arguments as in the theorem, the optimal contract necessarily involves $\Delta_{1}=z_{1}-\underline{w}, \Delta_{2}=z_{2}-\underline{w}$, and $p_{1}+p_{2}<1$. Moreover, it can also be proved that generically (that is, unless $k_{1}\left(z_{2}-\underline{w}\right)=k_{2}\left(z_{1}-\underline{w}\right)$ holds) incentives are focused on one of the goal levels: either $p_{1}=0$ and $p_{2}>0$; or $p_{1}>0$ and $p_{2}=0$. Therefore, the characteristics of the equilibrium contracts are the same as those in Theorem 1 for one out of the two goal levels. The same argument applies if there are more than two possible intensity levels or if the utility grows continuously from $z_{1}$ to $z_{2}$.

We discuss some potential policy implications of our analysis in Section 5, where we present three examples which are consistent with our theoretical setting. To guide this discussion, the next result (which proof follows from Theorem 1) states the effect of changes in the parameters $\underline{w}, z$, and $k$ on the goal-oriented agent's decisions at the equilibrium, as well as on the cut-off $\widehat{R}$ that characterizes the

\footnotetext{
${ }^{9}$ We have assumed that information about the agents' type is symmetric. What is important for Theorem 1 to hold is that a goal-oriented agent has the possibility to credibly provide evidence about his/her goal. For instance, an agent with an entrepreneurial project can inform principals about it, or a worker whose child can access the university can credibly highlight this fact to his/her potential employers.
} 
two sets of parameter values described in Theorem 1 .

Corollary 1. The comparative statics effects of

$\underline{w}, z$, and $k$ on a goal-oriented agent's equilibrium effort $e^{G}$, on his probability pe $e^{G}$ of triggering the extra utility $k$, and on the cut-off $\widehat{R}$, have the following signs:

\begin{tabular}{|c|c|c|c|c|c|}
\hline & $R<\widehat{R}$ & & $R \geqslant \widehat{R}$ & & \\
\hline & $e^{G}$ & $p e^{G}$ & $e^{G}$ & $p e^{G}$ & $\widehat{R}$ \\
\hline$\underline{w}$ & + & + & 0 & 0 & - \\
\hline $\bar{z}$ & - & - & 0 & 0 & + \\
\hline$k$ & + & + & + & + & - \\
\hline$R$ & + & + & + & + & . \\
\hline
\end{tabular}

We now move on to the case where the project value may depend on the principal's identity.

\subsection{Heterogeneous principals}

We now introduce the possibility that the principals may have different values associated to the success of the project: $R_{1}>R_{2}>\cdots>R_{n} \cdot{ }^{10}$ Given that goal-oriented agents are more appealing than standard agents to all the principals, the first question is to characterize the principals who will hire these agents at the equilibrium. Theorem 1 states that the goal-oriented agents' expected utility level increases with the return $R$, which suggests that the higher the return $R$, the more appealing is a goal-oriented agent to the principal. Lemma 4 confirms this intuition.

Lemma 4. If a principal with project value $R^{o}$ signs an optimal contract with a goal-oriented agent such that she obtains at least the same profits with the goal-oriented agent as with a standard agent, then any principal with project value $R>R^{o}$ prefers hiring the goal-oriented agent (instead of a standard one) under a contract that provides the agent with the same utility level than the contract signed with principal $R^{\circ}$.

An implication of Lemma 4 is that, at equilibrium, the goal-oriented agents will be hired by the principals whose project values in case of success are the highest ones, that is, principals 1 to $\mathrm{m}^{G}$. To attract the goal-oriented agents, these principals offer them a highenough level of utility so that the other principals (in particular, the "marginal" principal $m^{G}+1$ ) do not have incentives to hire a goaloriented instead of a standard one. As it is common in this type of setting with heterogeneous principals and agents, there are several equilibrium outcomes (in terms of levels of utility and profit), although the qualitative properties of these equilibria are similar in our framework. We are going to characterize one particular equilibrium, the one that corresponds to the most profitable equilibrium outcome for the principals.

Depending on the parameter values, the specifics of the equilibrium contract can differ. For simplicity, we are going to assume in Theorem 2 that $R_{m^{G}+1}$ is smaller than $\widehat{R}$, and that $R_{1}$ is not too large compared to $R_{m^{G}+1}$. We will then discuss what would happen in the other cases. The theorem uses some properties (Lemmas 1 and 3 ) that have been proved in the homogeneous case and that still hold when principals are heterogeneous.

Theorem 2. Under Assumption 1, if $R_{1}>R_{2}>\cdots>R_{n}, R_{m^{G}+1}<\widehat{R}$ and $R_{1}<\left(1+\sqrt{\frac{k}{k+z-\underline{w}}}\right) R_{m^{G}+1}$ then:
(a) Principal $i\left(i=1, \ldots, m^{G}\right)$ is matched with a goal-oriented agent under the dontract

$$
C^{G}\left(R_{i}\right)=\left(w=\underline{w}, \Delta=z-\underline{w}, p=\frac{1}{2(z-\underline{w})}\left(1+\sqrt{\frac{k}{k+z-\underline{w}}}\right) R_{m^{G}+1}\right),
$$

and principal $i\left(i=m^{G}+1, \ldots, n\right)$ is matched with a standard agent under the contract

$$
C^{S}\left(R_{i}\right)=\left(w=\underline{w}, \Delta=\frac{R_{i}}{2}, p=1\right) .
$$

(b) Principal $i$ obtains the following profit:

$$
\Pi\left(R_{i}\right)=\left\{\begin{array}{c}
\frac{(z-\underline{w}+k)}{4(z-\underline{w}) v}\left(1+\sqrt{\frac{k}{k+z-\underline{w}}}\right)\left(2 R_{i}-\left(1+\sqrt{\frac{k}{k+z-\underline{w}}}\right) R_{m^{G}+1}\right) R_{m^{G}+1}-\underline{w} \text { if } i \leqslant m^{G} \\
\frac{1}{4 v} R_{i}^{2}-\underline{w} \text { if } i>m^{G} .
\end{array}\right.
$$

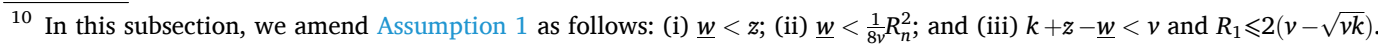


(c) Standard and goal-oriented agents obtain the following utility levels:

$$
\begin{aligned}
U^{S} & =\frac{1}{8 v} R_{i}^{2}+\underline{w} \text { when matched with principal } i>m_{G}, \\
U^{G} & =\frac{1}{8 v}\left(\frac{R_{m^{G}+1}}{z-\underline{w}}(k+z-\underline{w}+\sqrt{k(k+z-\underline{w})})\right)^{2}+\underline{w} .
\end{aligned}
$$

The first implication of Theorem 2 is that there is a positive assortative matching between principals and agents: the principals with most profitable projects hire the goal-oriented agents, that is, those agents who will exert higher effort. The higher the returns of a successful outcome, the more the principal benefits from hiring a goal-oriented agent instead of a standard one.

Second, the theorem highlights that in those partnerships involving a goal-oriented agent, the contract, the principal's profits and the goal-oriented agent's utility depend on the characteristic of the (marginal) principal who does not hire a goal-oriented agent, that is, on the principal whose project value is $R_{m^{G}+1}$. Due to their scarcity in the market, goal-oriented agents have market power because they can get offers from principals who hire standard agents. The best offer comes from the marginal principal, principal $m^{G}+1$. The better the offer that they can receive from this principal, the better the contract they sign with their employers at the equilibrium, thus the higher the utility they obtain, and the lower the employers' profit. As a consequence, if the population of goal-oriented agents is smaller (that is, $m^{G}$ is lower), then $R_{m^{G}+1}$ is higher and they obtain higher equilibrium utility level.

Third, as it is usual in moral hazard problems with limited liability, the contract and the utility of standard agents depend on the profitability of the project they work on. The more profitable the principal's project, the more she is interested in a high effort level and the higher the rent she provides the agent with. Thus, similar standard agents may enjoy different levels of utility at the equilibrium.

We notice that the comparative static exercises provided in Corollary 1 (except for those with respect to $R$ ) hold in the current setting with heterogeneous principals. Under the conditions stated in Theorem $2\left(R_{m^{G}+1}<\widehat{R}\right.$ and $\left.R_{1}<\left(1+\sqrt{\frac{k}{k+z-\underline{w}}}\right) R_{m^{G}+1}\right)$, the comparative statics related to the effort and to the probability of achieving the goal are the same as those for the case where $R<\widehat{R}$ holds. Both the effort level and the probability of triggering $k$ increase with $\underline{w}$ and $k$, and decrease with $z$.

Finally, we elaborate on the consequences of the assumptions about the principals' population in Theorem 2 . The main message does not change in the other cases, although the specifics of the contract differ. For instance, if $R_{m^{G}+1}<\widehat{R}$ but $R_{1}>(1+$ $\left.\sqrt{\frac{k}{k+z-\underline{w}}}\right) R_{m^{G}+1}$ holds, then the profitability of the project is so high for some principals that their optimal contract does not take into account the goal-oriented agent's participation constraint. The optimal contract only depends on the project value $R$. Still, the comparative exercises for the effort and the probability of achieving the goal are the same as before, unless the project value $R$ is very high, in which case only $k$ affects both $e^{G}$ and $p e^{G}$. Finally, if $R_{m^{G}+1}>\widehat{R}$ holds then the contract only depends on $k$ and not on $\underline{w}$ or $z$. In particular, if $R_{1}<\left(1+\sqrt{\frac{k}{k+z-\underline{w}}}\right) R_{m^{G}+1}$ holds then the contract is the same for all the principals who hire a goal-oriented agent: it does not depend on $R$ but on $R_{m^{G}+1}$. We now conclude this section by discussing an extension of the present analysis.

\subsection{When the goal is to avoid a penalty}

In the previous analysis, we have considered a setting where the goal constitutes an opportunity for some agents to obtain an extra utility. Reaching the goal (that is, receiving a salary higher than the trigger value) provides a goal-oriented agent with an extra utility compared to a standard agent.

We could also consider settings where obtaining a certain amount of money does not open up an opportunity to improve on the agent's utility, but it is rather a requirement for the agent in order to avoid a penalty. For instance, an indebted individual may experience economic or judicial consequences if he does not repay his debt. The trigger in this case corresponds to the level of the debt: if the individual does not obtain the trigger value $z$, he experiences a disutility $k$. We can call such an individual a "loss-avoidance agent." His utility function is identical to that of a standard agent for monetary payment levels lying above $z$ but the utility level is lowered by $k$ if the monetary payment lies below $z .{ }^{11}$ As we made it clear in the previous subsections, a goal-oriented agent is more appealing to the principals than a standard agent because of two reasons: the additional incentives due to the possibility of reaching the

\footnotetext{
${ }^{11}$ Although the models are quite different, there are some similarities between the idea that agents experience diminishing utility if they do not reach their goal and the idea of loss-averse agents (Kahneman \& Tversky, 1979; Tversky \& Kahneman, 1991, 1992). In both cases, the shape of the agent's utility function changes at some reference income level. For an analysis of optimal contracts with loss-averse agents, see for instance De Meza and Webb (2007) and Herweg et al. (2010).
} 
goal, and the higher utility obtained by a goal-oriented agent. In terms of incentives, a loss-avoidance agent is similar to a goal-oriented agent. The existence of a kick at the trigger value provides either type of agent with an extra incentive if $w<z \leqslant w+\Delta$ and $p>0$ hold. However, a loss-avoidance agent may be more "expensive" not only than a goal-oriented agent but also than a standard agent. Indeed, given that they experience a lower utility level than standard agents for monetary payment levels lying below $z$, loss-avoidance agents may require a higher average salary. Therefore, it is a priori not clear whether a loss-avoidance agent is more or less appealing to a principal than a standard agent.

If we consider a market where loss-avoidance agents coexist with standard agents (and the number of standard agents is large) then, as in a market with goal-oriented agents, the positive effect due to the extra incentives is stronger the higher the value $R$ associated to success. Therefore, if $R$ is high enough, a principal prefers a loss-avoidance agent to a standard agent. What are the implications of this property for the equilibrium contracts?

First, if the principals are homogeneous (as in subSection 4.1) and $R$ is high enough then, at equilibrium, the principals will compete for the loss-avoidance agents. Given that these agents' utility level is determined by the degree of competition (and not by their outside option) the equilibrium contracts (in this case) are identical to those stated in Theorem 1 . On the other hand, if $R$ is low, then these agents are not appealing to the principals and will not be hired at equilibrium. ${ }^{12}$ Second, if the principals are heterogeneous in the values associated with the success of the project (as in subSection 4.2) then Lemma 4 still holds, which implies that, if some lossavoidance agents are hired in the market, they are matched to the principals with the highest project values. Depending on the value of $R$ for the top principals, it may happen that the principals compete for the loss-avoidance agents (this is the case if the project value $R$ for the "marginal" principal is high enough), in which case Theorem 2 still applies. But, it may also happen that only a few lossavoidance agents (or even none of them) are hired by the principals with the highest project values. In this case, these principals can offer a contract where a loss-avoidance agent's participation constraint is not characterized by competition forces but by his outside option.

\section{Policy interventions}

In this section, we describe three examples that are consistent with our analytical setting. In the three examples, obtaining a highenough payment allows some agents to achieve their goal. We discuss the implications of several policy interventions making use of the comparative statics results provided in Corollary 1 and in the discussion following Theorem 2. The purpose of the examples is to illustrate the effects driven by goal-oriented agents in some very stylized situations where other important features, which we ignore here, may be present.

To simplify the explanations, we consider situations where $R_{m^{G}+1}<\widehat{R}$ and $R_{1}<\left(1+\sqrt{\frac{k}{k+z-\underline{w}}}\right) R_{m^{G}+1}$ (or $R<\widehat{R}$ in case of an homogeneous technology) are satisfied. The discussion for the other cases is similar, unless the project value of the "marginal" principal (that is, $R_{m^{G}+1}$ ) is larger than $\widehat{R}$, a situation in which the contracts signed with the goal-oriented agents only depend on $k$.

\subsection{Entrepreneurship}

Consider the case where the goal of some agents is to become entrepreneurs. Each of these agents has an idea that can yield profits in a different market. However, he needs some capital to develop his business idea, and this is the reason why he works in the labor market considered here.

Indeed, becoming an entrepreneur requires a certain investment level. The idea is risky, in that it yields a positive outcome only with some probability. To provide a simple framework we assume, following Bester and Hellwig (1987) and Tirole (2010, chap. 3), that if the idea is carried out, the entrepreneur can take one of two possible decisions. The venture yields positive net return only if the correct decision is taken. The alternative decision yields negative net present value but provides private benefits to the entrepreneur. In this framework, due to the entrepreneur's moral hazard problem, he only takes the correct decision if he keeps enough shares of the new venture. This puts a constraint on the shares that the entrepreneur can offer to a financier, hence, to the amount of money that he can borrow. In other words, because of the moral hazard problem concerning the entrepreneur's decision, there is a minimum amount of money that cannot come from external financiers.

The model parameters can be interpreted as follows. The trigger value $z$ corresponds to the minimum amount of money that the agent needs to invest in the venture in order to attract a loan and to become an entrepreneur. The extra utility $k$ models two complementary aspects. First, it models the expected net profit that the agent obtains if he starts the venture. Second, it may also relate to behavioral aspects of entrepreneurship, as studied in Gilad and Levine (1986). According to their contribution (the "pull theory" of entrepreneurship), individuals are attracted into entrepreneurial activities seeking independence and self-fulfillment (in addition to wealth).

Policy makers often have incentives to promote entrepreneurial activities. We now discuss interventions in the labor market that can indirectly affect the entrepreneurial activity. We also show that a policy intervention targeting the entrepreneurial activity may

\footnotetext{
12 Depending on the other model parameters, it could be the case that any principal (that is, any $R$ ) prefers a loss-avoidance agent to a standard agent or that (because of the constraint that the effort level cannot be larger than one) any principal prefers a standard agent to a loss-avoidance agent.
} 
have indirect effects on the labor market.

Let us first consider an increase in the base salary $\underline{w}$. Increasing the base salary yields both a higher effort level exerted by goaloriented agents and a higher probability that the bonus be awarded to these agents in case of success. This second effect translates in turn into an increase in the probability that these agents become entrepreneurs (as measured by pe) and that they enjoy extra utility $k$. Moreover, this increase in the base salary has an indirect consequence: it decreases the threshold value $\widehat{R}$, which reinforces the other positive effect on entrepreneurship.

It is worth mentioning that a policy that increases $\underline{w}$ has also redistributive and efficiency effects. Increasing the base salary has ceteris paribus a positive effect for all the agents and a negative effect for all the principals. There is an efficiency effect as the agents' incentives and optimal effort level increase, which makes the equilibrium effort closer to the first-best level and thus increases total welfare.

A second intervention might consist in changing the trigger value $z$. In terms of the effort supplied and the likelihood to become an entrepreneur, a decrease in $z$ is equivalent to an increase in $\underline{w}$. Such a decrease in $z$ can be achieved, for instance, by using a policy that provides a subsidy to new entrepreneurs, or that decreases the total investment needed to carry out the project, for instance by softening the administrative and legal burden. Such a policy (targeting the entrepreneurial activity) increases the goal-oriented agents' motivation to exert effort in their current job, which in turn positively impacts principals' profit. Moreover, it also increases these agents' utility level because they are more likely to become entrepreneurs. One must however keep in mind that a subsidy scheme that would decrease $z$ might need to be funded by the government. Therefore, the positive effect on the goal-oriented agents' utility needs to be traded off with the cost resulting from the use of public funding: The effect on total welfare depends on the extra cost resulting from the use of public funding.

Finally, the government can change the taxes and fees paid by entrepreneurs (or self-employed people), affecting the expected income of these activities, that is, the extra utility $k$. As we have seen in the comparative static results, increasing $k$ always has a positive effect on the goal-oriented agents' effort and the probability of becoming an entrepreneur. Therefore, reducing the charges to be paid by entrepreneurs not only increases the number of entrepreneurs but it also has an indirect positive effect on the markets where these individuals worked as agents.

\subsection{Buying an asset with persistent benefits}

The additional motivation of the agent can result from buying a certain good or service. Consider for simplicity that the agent has limited access to the loan market. He may have the will to get college education but lacks the monetary resources to do it. Alternatively, he may want to send his child to college (Becker, 2009). In this case, the trigger value $z$ corresponds to the minimum amount of money the agent needs to finance college education, and $k$ corresponds to the extra salary and utility that he derives once he (or his child) obtains a college degree. The same idea can apply to the access to secondary education in many developing countries, where financing is a challenge for many families.

In another example with similar characteristics, the agent may be willing to buy some particularly relevant good characterized by long-lasting benefits, like a house. In this case, the extra utility $k$ may correspond to the pleasure and utility that the agent gets from owning such a house. As previous literature suggests (see, e.g., Flavin \& Yamashita, 2002), many individuals have strong motivations for home ownership: getting protection against future negative shocks, using ownership as a commitment device to ensure some monetary savings, or social achievement. ${ }^{13}$ In this setting, a policy intervention may be designed to increase the likelihood that the goal is achieved, because the policy maker's goal may be to ensure that the agents invest in education or housing.

The effect of a change in the base salary $\underline{w}$ or of a policy that changes the trigger value $z$ is similar as in the previous example. Here, a decrease in $z$ can be achieved, for instance, by subsidizing the people newly registered for college education, or through a policy decreasing either the frictions in the credit market or the administrative and legal burden (which may prevail in the housing market). As in the previous example, policies implemented in the labor market produce externalities on the education level or on the acquisition of certain goods. Similarly, policies targeting the access to education or housing induce externalities in the labor market.

\subsection{Aspiration-based trigger}

Another example that is consistent with our analysis is based on behavioral motives. The agent's additional motivation can result from reaching an aspiration-based reference compensation level. Specifically, some agents may exhibit extra utility based on individual aspirations. As explained in the literature (see Ray, 1998, among other references), aspirations are ambitions of achieving something which may or may not be achievable in reality. In our setting, up to a certain payment level, a goal-oriented agent might not experience extra utility as he feels like his ambition is not being fulfilled. Once his compensation is at least equal to this threshold level, the agent experiences some extra utility, as he thinks that his ambition has been satisfied.

In this case, the trigger value $z$ corresponds to the aspiration-based reference payment level, and $k$ corresponds to the extra utility

\footnotetext{
${ }^{13}$ If the agent has access to some credit market, the trigger value $z$ is the minimum amount required to obtain a loan. This minimum amount is required for reasons that differ from the moral hazard problem identified in Section 5.1. In the case of housing, the financial institutions only lend a share of the price of the house because there is a chance that the individual cannot repay the loan (due to unemployment or some health hazard) and there is also a chance that the market price of the house decreases, which could prevent the financier to recover the full loan in case the individual fails to make mortgage payments.
} 
that he derives once he considers that his ambition has been fulfilled. Using the discussion provided by Génicot and Ray (2020), here aspirations are achievable, and the corresponding utility does not depend on the excess of outcome over the aspiration level. Our analysis shares a conclusion that is often reached in this literature: an increase in the level of aspiration (measured in terms of $z$ ) may prove to be detrimental to the agent, as the agent will receive the bonus in case of success with lower probability. This differs from a setting where too high aspirations would be detrimental as they may create frustration (and thus disutility). ${ }^{14}$ In terms of public policies, changing the base salary $\underline{w}$ or impacting the trigger level $z$ have the same consequences as above. While modifying the base salary might be implemented by using a fairly straightforward policy, impacting z might be less straightforward. Some policies aimed at changing the reference point have been discussed in the literature on aspirations and economic behaviors (we refer to La Ferrara, 2019, for an extensive discussion on this point).

\section{Conclusion}

In a market environment where goal-oriented agents coexist with standard ones, we analyze how the heterogeneity in the agents' population affects the equilibrium outcome in terms of matching and contracts. Compared to standard agents, goal-oriented agents derive extra utility from achieving a high-enough monetary payoff (the "trigger") as this allows them to fulfill an individual goal. We obtain several interesting results. First, in any equilibrium contract, a goal-oriented agent reaches the trigger with a positive probability. However, a successful outcome does not ensure that goal-oriented agents achieve their ambitions: if the trigger is too high, then the bonus is only paid with some probability. We also show that, at the equilibrium, goal and monetary incentives are complementary: goal-oriented agents receive stronger monetary incentives than standard agents. Finally, we highlight that the matching between principals and agents is positive assortative. These results have in turn interesting and important policy implications in relevant situations. Specifically, we highlight how the presence of goal-oriented agents creates a wedge between different markets, which results in important feedback effects of policy interventions. This contribution stresses the importance for contractual and policy designs of acknowledging the existence of goal-oriented agents, and calls for further research along this line.

In this paper, we assume that information about the agents' type is symmetric. However, let us stress that what is important for our main result to hold is that a goal-oriented agent can credibly provide evidence about his/her goal. For instance, an agent with an entrepreneurial project can inform principals about it, or a worker whose child can access the university can credibly highlight this fact to his potential employers. Indeed, when there are market interactions, goal and monetary incentives are complementary and goaloriented agents have an incentive to provide the information about their type. Given that the principals compete for contracting with them, the goal-oriented agents obtain a higher equilibrium utility level, which translates into higher incentives.This is in contrast to an environment without market interaction, where goal and monetary incentives are substitutes, and as such goal-oriented agents would have an incentive to hide their type.

\section{Appendix A}

Proof of Lemma 1: The Pareto optimal contract between a principal and a standard agent whose outside utility is $U^{o}=0$ solves the following program:

$$
\begin{array}{r}
\operatorname{Max}_{(w, \Delta, p)}\{e R-e p \Delta-w\} \\
\text { s.t. } \quad w+e p \Delta-\frac{v}{2} e^{2} \geqslant 0 \\
e=\frac{p \Delta}{v} \\
w \geqslant \underline{w}, \quad p \geqslant 0, \quad p \leqslant 1
\end{array}
$$

where $p$ and $\Delta$ are always considered as a product, except for the constraint that $p \in[0,1]$. Therefore, any optimal solution is equivalent to a solution where $p=1$ and the endogenous variables are $(w, \Delta)$. Since the standard agent's "reservation utility" is zero, the problem corresponds to the classical case with limited liability and the solution is well known: given that $R \geqslant 2 \sqrt{2 v \underline{U}}=0$ holds, one optimal contract is $\left(w=\underline{w}, \Delta=\frac{R}{2}\right)$, the agent's effort level is $e=\frac{R}{2 v} \in[0,1](R \leqslant 2 v$ by Assumption 1$)$, and the principal's profit is $\frac{R^{2}}{4 v}-\underline{w}$. The contracts described in the lemma are equivalent to $\left(w=\underline{w}, \Delta=\frac{R}{2}, p=1\right)$.

Proof of Lemma 3: We prove the lemma in several steps.

Step 1. In an optimal contract, $w<z$ is satisfied.

\footnotetext{
14 This setting is different from situations where the extra utility results from choosing the action that the agent considers as the right one, or from situations where the extra utility results from doing the task that the agent considers as consistent with the mission of his organization (see for instance Besley \& Ghatak, 2005, Auriol \& Brilon, 2014, who analyze a setting where some workers care about the mission of their organizations in the nonprofit sector).
} 
Proof of step 1 . We prove it by contradiction. Suppose that the equilibrium contract sets $w \geqslant z$ and denote $U^{G^{*}}$ the goal-oriented agent's utility under the contract. It is Pareto-efficient, that is, it solves the following program:

$$
\begin{array}{r}
\operatorname{Max}_{(w, \Delta, p)}\{e R-e p \Delta-w\} \\
\text { s.t. } \quad w+k+e p \Delta-\frac{v}{2} e^{2}=U^{G^{*}} \\
e=\frac{p \Delta}{v} \\
w \geqslant z \\
\Delta \geqslant 0, \quad p \geqslant 0, \quad p \leqslant 1 .
\end{array}
$$

The variables $p$ and $\Delta$ are always considered as a product, except for $p \in[0,1]$. Therefore, if $p$ was smaller than one, we could substitute $p$ by 1 and $\Delta$ by $\Delta^{\prime}=p \Delta$ and the program would be equivalent. Hence, we take $p=1$ and using $e=\frac{1}{v} \Delta$ the program is equivalent to $w=$ $U^{G^{*}}-k-\frac{1}{2 v} \Delta^{2}$ and

$$
\operatorname{Max}_{\Delta}\left\{\frac{1}{v} R \Delta-\frac{1}{2 v} \Delta^{2}-U^{G^{*}}+k\right\} \quad \text { s.t. } \quad U^{G^{*}}-k-\frac{1}{2 v} \Delta^{2} \geqslant z \quad(\gamma) \quad \Delta \geqslant 0 . \quad(\sigma)
$$

The first-order condition (FOC) of the Lagrangian with respect to $\Delta$ is:

$$
\frac{\partial}{\partial \Delta}=\frac{1}{v} R-\frac{1}{v} \Delta-\frac{1}{v} \Delta \gamma+\sigma=0
$$

which implies $\gamma>0$ because $\Delta \geqslant R$ would yield negative profits, and the Kuhn-Tucker (KT) conditions imply $\sigma \geqslant 0$ necessarily. Hence, the candidate contract is $\Delta=\sqrt{2 v\left(U^{G^{*}}-k-z\right)}$ and $w=z$. This solution is only defined if $\sigma \geqslant 0$, that is, $U^{G^{*}} \geqslant k+z$ holds. The principal's profit under the previous contract is

$$
\Pi\left(U^{G^{*}}\right)=R \sqrt{\frac{2}{v}\left(U^{G^{*}}-k-z\right)}-2 U^{G^{*}}+2 k+z .
$$

The utility level $U^{G^{*}}$ is characterized at the equilibrium. According to Lemma 2, it satisfies $\Pi\left(U^{G^{*}}\right)=\Pi^{S^{*}}=\frac{1}{4 v} R^{2}-\underline{w}$, that is:

$$
R \sqrt{\frac{2}{v}\left(U^{G^{*}}-k-z\right)}-2 U^{G^{*}}+2 k+z=\frac{1}{4 v} R^{2}-\underline{w}
$$

which, denoting $Q \equiv \frac{R}{\sqrt{v}}$ and $Y \equiv U^{G^{*}}-k$, we write as

$$
f(Y) \equiv \sqrt{2} Q \sqrt{Y-z}-2 Y+z+\underline{w}-\frac{1}{4} Q^{2}=0 .
$$

Note that $f^{\prime}(Y)>0$ if $Y<z+\frac{1}{8} Q^{2}$ and $f^{\prime}(Y)<0$ if $Y>z+\frac{1}{8} Q^{2}$. The minimum value of $Y$ is $Y=z$ (because $\underline{U}^{G} \geqslant k+z$ ) where we have $f(z)<0$. Then, the maximum value of $f(Y)$ is reached for $Y=z+\frac{1}{8} Q^{2}$. Finally, $f\left(Y=z+\frac{1}{8} Q^{2}\right)=\sqrt{2} Q \sqrt{\frac{1}{8} Q^{2}}-2 z-\frac{1}{4} Q^{2}+z+$ $\underline{w}-\frac{1}{4} Q^{2}=-z+\underline{w}<0$. Therefore, equation (2) has no solution. Hence, we conclude that the equilibrium contract $C^{G}$ cannot include a payment $w \geqslant z$.

Step 2. Any optimal contract $(w, \Delta, p)$ stipulates $p>0$.

Proof of Step 2. If $p=0$ the agent exerts effort level $e=0$, in which case the principal's profit is negative.

Step 3. Any contract $(w, \Delta, p)$ with $w<z$ and $\Delta<z-w$ is dominated by $\left(w, \Delta^{\prime}=z-w, p^{\prime}=p \frac{\Delta}{z-w+k}\right)$, that is, the principal induces the extra motivation with some positive probability. Hence, at the equilibrium contract $\Delta \geqslant z-w$ is satisfied.

Proof of Step 3. A contract $(w, \Delta, p)$ with $w<z$ and $\Delta<z-w$ yields:

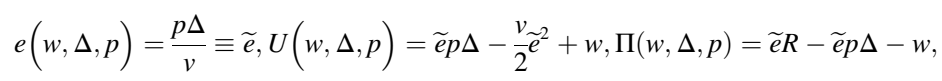

where $\widetilde{e} \leqslant 1$ by Assumption 1 . Now consider the contract $\left(w=w, \Delta^{\prime}=z-w, p^{\prime}=p \frac{\Delta}{z-w+k}\right)$ and notice that $p^{\prime}<p \frac{z-w}{z-w+k}<p$ are satisfied. Under the new contract, the agent still exerts effort $\widetilde{e}$ (because $\left.p^{\prime}(z-w+k)=p \Delta\right)$ and he gets the same utility level as under the initial contract:

$$
U\left(w, z-w, p^{\prime}\right)=\widetilde{e} p^{\prime}(k+z-w)-\frac{v_{\widetilde{e}}}{2}+w=\widetilde{e} p \Delta-\frac{v^{2}}{2}+w .
$$


Moreover, the principal prefers the new contract:

$$
\Pi\left(w, z-w, p^{\prime}\right)=\widetilde{e} R-\widetilde{e} p \Delta \frac{(z-w)}{(k+z-w)}-w>\widetilde{e} R-\widetilde{e} p \Delta-w .
$$

Therefore, the initial contract $(w, \Delta, p)$ is not Pareto optimal.

Step 4. Any contract $(w, \Delta, p)$ with $w \in(\underline{w}, z)$ and $\Delta \geqslant z-w$ is dominated by (or equivalent to, if the agent's effort level is equal to one) the contract $\left(w=\underline{w}, \Delta^{\prime}, p\right)$ with $\Delta^{\prime}=\Delta+\frac{w-w}{p e}$, where $\widetilde{e}=\min \left\{\frac{p(\Delta+k)}{v}, 1\right\}$. Hence, at the equilibrium contract $w=\underline{w}$.

Proof of Step 4: If $w \in(\underline{w}, z), \Delta \geqslant z-w$ and $p>0$ we have:

$$
e(w, \Delta, p)=\min \left\{\frac{p(\Delta+k)}{v}, 1\right\} \equiv \widetilde{e}, U(w, \Delta, p)=w+\widetilde{e} p \Delta+\widetilde{e} p k-\frac{v^{2}}{2}, \Pi(w, \Delta, p)=\widetilde{e} R-\widetilde{e} p \Delta-w
$$

Consider now $\left(w=\underline{w}, \Delta^{\prime}=\Delta+\frac{w-w}{p e}, p\right)$. If the agent exerts the same effort level $\widetilde{e}$ he will get the same utility level and the principal will obtain the same profit. If $\widetilde{e}<1$ holds, the agent increases his effort level (because $\Delta^{\prime}>\Delta$ ) under the new contract, and he is better off. Moreover, since the profit is increasing in the agent's effort level, the principal obtains higher profits than under the initial contract.

Proof of Theorem 1: The agent's effort level is $e=\min \left\{\frac{p(\Delta+k)}{v}, 1\right\}$. We are going to compute the optimal contract where we substitute $e$ by $\frac{p(\Delta+k)}{v}$. For the contract that we will characterize, the agent's effort level is indeed lower than one, hence it is also optimal under the true equality $e=\min \left\{\frac{p(\Delta+k)}{v}, 1\right\}$. If $e=\frac{p(\Delta+k)}{v}$ the agent's expected utility is:

$$
U=\underline{w}+e p(\Delta+k)-\frac{v}{2} e^{2}=\underline{w}+\frac{p^{2}(\Delta+k)^{2}}{2 v} .
$$

Substituting the optimal agent's effort level in the expression of the principal's profit, and taking into account that her equilibrium profit must be $\frac{1}{4 v} R^{2}-\underline{w}$, the equilibrium contract is the solution to the following program:

$$
\begin{array}{r}
\operatorname{Max}_{\Delta, p}\left\{\frac{p^{2}(\Delta+k)^{2}}{2 v}+\underline{w}\right\} \\
\text { s.t. } \quad \frac{p(\Delta+k)}{v}(R-p \Delta)-\underline{w} \geqslant \frac{1}{4 v} R^{2}-\underline{w} \\
\Delta \geqslant z-\underline{w}, 1 \geqslant p,
\end{array}
$$

which is equivalent to:

$$
\begin{array}{rr}
\operatorname{Max}_{\Delta, p}\left\{p^{2}(\Delta+k)^{2}+2 \underline{v w}\right\} \\
\text { s.t. } 4 p(\Delta+k)(R-p \Delta)-R^{2}=0 \quad(\lambda) \\
\Delta \geqslant z-\underline{w} \\
1 \geqslant p
\end{array}
$$

and FOCs are

$$
\begin{gathered}
\frac{\partial}{\partial \Delta}=2 p^{2}(\Delta+k)-\lambda 4 p(2 p \Delta-R+k p)+\alpha=0 \\
\frac{\partial}{\partial p}=2 p(\Delta+k)^{2}+\lambda 4(\Delta+k)(R-2 p \Delta)-\gamma=0 .
\end{gathered}
$$

There are three possible cases.

(i) If $\alpha>0$ and $\gamma=0$, then $\Delta=z-\underline{w}$. Constraint $(\lambda)$ becomes $4 p(z-\underline{w}+k)(R-p(z-\underline{w}))-R^{2}=0$. Solving the equation in $p$ yields two solutions: $p=\frac{1}{2(z-\underline{w})}\left(1 \pm \frac{\sqrt{k}}{\sqrt{z-\underline{w}+k}}\right) R$. The smallest root is not a candidate because it would yield $R-2 p \Delta=\frac{\sqrt{k}}{\sqrt{z-\underline{w}+k}} R>0$, 
in which case (3b) cannot hold. Therefore,

$$
p=\frac{1}{2(z-\underline{w})}\left(1+\frac{\sqrt{k}}{\sqrt{z-\underline{w}+k}}\right) R
$$

From (3b) we have $\lambda=-\frac{2 p(\Delta+k)^{2}}{4(\Delta+k)(R-2 p \Delta)}=\frac{(\sqrt{z-\underline{w}+k}+\sqrt{k})(z-\underline{w}+k)}{4(z-\underline{w}) \sqrt{k}}>0$. Also, (3a) implies $\alpha=\frac{(\sqrt{z-\underline{w}+k}+\sqrt{k})^{2}(z-\underline{w}+k)}{2(z-\underline{w})^{2} \sqrt{z-\underline{w}+k}}$ $\left(\frac{1}{\sqrt{k}}\left(\frac{\sqrt{k}}{\sqrt{z-\underline{w}+k}}+k \frac{1}{2(z-\underline{w})}\left(1+\frac{\sqrt{k}}{\sqrt{z-\underline{w}+k}}\right)\right)-\frac{1}{\sqrt{z-\underline{w}+k}}\right) R^{2}$. Therefore, $\alpha>0$ if and only if $\left.\frac{1}{\sqrt{k}}\left(k \frac{1}{2(z-\underline{w})}\left(1+\frac{\sqrt{k}}{\sqrt{z-\underline{w}+k}}\right)\right)\right\rangle$, which always holds. Finally, constraint $(\gamma)$ requires

$$
R \leqslant \frac{2(z-\underline{w}) \sqrt{z-\underline{w}+k}}{\sqrt{z-\underline{w}+k}+\sqrt{k}}=2(z-\underline{w}+k-\sqrt{k(z-\underline{w}+k)})
$$

which is the condition for the contract identified in this case to be a candidate. The agent's effort level under the contract is

$$
e=\frac{p(\Delta+k)}{v}=\frac{1}{2 v(z-\underline{w})}(z-\underline{w}+k+\sqrt{k(z-\underline{w}+k)}) R=\frac{\sqrt{z-\underline{w}+k}}{2 v(\sqrt{z-\underline{w}+k}-\sqrt{k})} R .
$$

The effort level is increasing in $R$. At the upper limit of the interval, that is, when $R=2(z-\underline{w}+k-\sqrt{k(z-\underline{w}+k)})$ the effort level is $e=\frac{1}{v}(z-\underline{w}+k)$ which is smaller than one by Assumption 1 .

The agent's utility under the contract is

$$
U=\frac{p^{2}(\Delta+k)^{2}}{2 v}+\underline{w}=\frac{1}{8 v}\left(\frac{1}{(z-\underline{w})}((z-\underline{w}+k)+\sqrt{k(z-\underline{w}+k)}) R\right)^{2}+\underline{w} .
$$

(ii) If $\alpha=0$ and $\gamma>0$, then $p=1$ holds. Also, constraint ( $\lambda$ ) yields $4(\Delta+k)(R-\Delta)-R^{2}=0$. This equation has two solutions in $\Delta$ : $\Delta=\frac{R-k \pm \sqrt{k(2 R+k)}}{2}$. The smallest root cannot be a candidate because in that case we would have $2 p^{2}(\Delta+k)>0$ and $4 p(2 p \Delta-R+k p)=$ $-4(\sqrt{k(2 R+k)})<0$ which is not compatible with equation (3a). Therefore, the candidate bonus in this region is

$$
\Delta=\frac{1}{2}(R-k+\sqrt{k(2 R+k)})
$$

From (3a) and $\alpha=0$ we deduce $\lambda=\frac{2 p^{2}(\Delta+k)}{4 p(2 p \Delta-R+k p)}=\frac{R+k+\sqrt{k(2 R+k)}}{4 \sqrt{k(2 R+k)}}$. Moreover, from (3b) we have

$$
\frac{1}{2}(R+k+\sqrt{k(2 R+k)})^{2}+\frac{R+k+\sqrt{k(2 R+k)}}{2 \sqrt{k(2 R+k)}}(R+k+\sqrt{k(2 R+k)})(k-\sqrt{k(2 R+k)})-\gamma=0, \text { hence } \gamma=\frac{1}{2}(R+k+\sqrt{k(2 R+k)})^{2}
$$
$\frac{k}{\sqrt{k(2 R+k)}}>0$ holds. Finally, equation $(\alpha)$ requires $\Delta \geqslant z-\underline{w}$, i.e., $\frac{1}{2}(R-k+\sqrt{k(2 R+k)}) \geqslant z-\underline{w}$, or

$$
R \geqslant 2(z-\underline{w}+k-\sqrt{k(z-\underline{w}+k)}) .
$$

The agent's effort level under the contract is

$$
e=\frac{p(\Delta+k)}{v}=\frac{1}{2 v}(R+k+\sqrt{k(2 R+k)}) .
$$

After some calculations, one can check that $e \leqslant 1$ if and only if $R \leqslant 2(v-\sqrt{v k})$, which holds due to Assumption 1 .

The agent's utility under the contract is 


$$
U=\frac{p^{2}(\Delta+k)^{2}}{2 v}+\underline{w}=\frac{1}{8 v}(R+k+\sqrt{k(2 R+k)})^{2}+\underline{w} .
$$

(iii) If $\alpha>0$ and $\gamma>0$ then $p=1$ and $\Delta=z-\underline{w}$. This case coincides with the limit case of (i) and (ii) and the conditions (for a candidate solution) are identical. Condition $(\lambda)$ is satisfied if $R=2(z-\underline{w}+k-\sqrt{k(z-\underline{w}+k)})$.

(iv) If $\alpha=0$ and $\gamma=0$ then easy calculations show that (3a) and (3b) cannot hold simultaneously if $p>0$, hence there is no candidate solution in this case.

Proof of Lemma 4: Denote $\underline{U}^{G}$ the utility level obtained by the goal-oriented agent if he signs an optimal contract with a principal potentially generating value $R$ and let $(\Delta, p)$ be the contract. The contract $(\Delta, p)$ solves the following program $(P P)$ :

$$
\begin{array}{rr}
\operatorname{Max}_{\Delta, p}\left\{\frac{p(\Delta+k)}{v}(R-p \Delta)-\underline{w}\right\} \\
\text { s.t. } \quad \frac{p^{2}(\Delta+k)^{2}}{2 v}+\underline{w} \geqslant \underline{U}^{G} \quad(\lambda) \\
\Delta \geqslant z-\underline{w} \quad(\alpha) \\
1 \geqslant p \quad(\gamma)
\end{array}
$$

and the FOC with respect to $\Delta$ is:

$$
\frac{\partial}{\partial \Delta}=\frac{1}{v} p(R-2 p \Delta-k p)+\lambda \frac{1}{v} p^{2}(\Delta+k)+\alpha=0
$$

Define $\delta(R)$ as the difference between the profit obtained by a principal whose project's value is $R$ under contract ( $p, \Delta)$ and the profit obtained with a standard agent, i.e.,

$$
\delta(R) \equiv \frac{1}{v} p(\Delta+k)(R-p \Delta)-\underline{w}-\left(\frac{1}{4 v} R^{2}-\underline{w}\right) .
$$

Using the envelop theorem, we have $\frac{\partial \delta}{\partial R}=\frac{1}{v} p(\Delta+k)-\frac{1}{2 v} R$. Then, $\frac{\partial \delta}{\partial R}>0$ if and only if $R-2 p(\Delta+k)<0$. We rewrite equation (4) as $R-2 p \Delta-2 k p=-k p-\lambda p(\Delta+k)-\frac{1}{p} \alpha v$; hence $\frac{\partial \delta}{\partial R}>0$. This implies that the difference in profits increases with $R$.

Therefore, suppose that a principal whose project generates value $R^{o}$ (denote her $R^{o}$ ) hires a goal-oriented agent and consider a principal with $R>R^{o}$ (denote her $R$ ). Then, we show that, at the equilibrium, it is necessarily the case that principal $R$ also hires a goaloriented agent.

If $\underline{U}^{G}$ is the utility level obtained by the goal-oriented agent in the contract with principal $R^{o}$, then $\underline{U}^{G}$ is lower than or equal to $\underline{U}$ satisfying that $R^{o}$ 's profits related to $\underline{U}$ are equal to $\frac{1}{4 v} R^{2}-\underline{w}$. However, if principal $R$ would be hiring a standard agent, she would be ready to pay the goal-oriented agent a higher level of utility (as she would obtain higher profits). Therefore, this could not be an equilibrium, which proves the lemma.

Proof of Theorem 2. Denote $\underline{U}^{G}$ the utility level obtained by the goal-oriented agent under the contract with principal $R_{m^{G}+1}$ that would make this principal indifferent between hiring him and hiring a standard agent. Under this contract, the agent obtains the utility level $U^{G}$ characterized in Theorem 1 . This is the utility level of any goal-oriented agent in the market. Since we assume that $R_{m^{G}+1}<$ $\widehat{R}=2(z-\underline{w}+k-\sqrt{k(z-\underline{w}+k)})$ we have $\underline{U}^{G}=\frac{1}{8 v}\left(\frac{R_{m^{G}+1}}{z-\underline{w}}(k+z-\underline{w}+\sqrt{k(k+z-\underline{w})})\right)^{2}+\underline{w}$.

For any principal $R$ (from $R_{1}$ to $R_{m^{G}}$ ), her contract $(\Delta, p)$ solves the same program $(P P)$ as in the proof of Lemma 4. The FOCs of $(P P$ ) are:

$$
\begin{aligned}
& \frac{\partial}{\partial \Delta}=\frac{1}{v} p(R-2 p \Delta-k p)+\lambda \frac{1}{v} p^{2}(\Delta+k)+\alpha=0 \\
& \frac{\partial}{\partial p}=\frac{1}{v}(\Delta+k)(R-2 p \Delta)+\lambda \frac{1}{v} p(\Delta+k)^{2}-\gamma=0 .
\end{aligned}
$$

We consider $\lambda>0$, as there is no candidate with $\lambda=0$.

$$
\begin{aligned}
& \text { (i) If } \alpha>0 \text { and } \gamma=0 \text {, then } \Delta=z-\underline{w} \text {. Moreover, binding constraint }(\lambda) \text { yields } \frac{p^{2}(\Delta+k)^{2}}{2 v}+\underline{w}= \\
& \frac{1}{8 v}\left(\frac{R_{m^{G}+1}}{z-\underline{w}}(k+z-\underline{w}+\sqrt{k(k+z-\underline{w})})\right)^{2}+\underline{w} \text {, that is, }
\end{aligned}
$$




$$
p=\frac{1}{2(z-\underline{w})}\left(1+\sqrt{\frac{k}{k+z-\underline{w}}}\right) R_{m^{G}+1} .
$$

We notice that $p<1$ given that $R_{m^{G}+1}<\widehat{R}$ holds. From (5b), we have $\lambda=\frac{2 p \Delta-R}{p(\Delta+k)}$. Therefore, $\lambda>0$ if and only if $R-2 p \Delta<0$, i.e., $R<$ $\left(1+\sqrt{\frac{k}{k+z-\underline{w}}}\right) R_{m^{G}+1}$ which holds according to the assumptions. Finally, from (5a) we have $\alpha=\frac{1}{v} p(k p+2 p \Delta-R)-\lambda \frac{1}{\nu} p^{2}(\Delta+k)$. Then, $\alpha>0$ if and only if $k p+2 p \Delta-R>2 p \Delta-R$ which always holds. Therefore, this solution is a candidate. Principal $i$ hiring a goal-oriented agent gets the profit $\Pi\left(R_{i}\right)=\frac{p(\Delta+k)}{v}(R-p \Delta)-\underline{w}$, which, after substituting for the optimal contract, yields the expression provided in the theorem.

(ii) If $\alpha=0$ and $\gamma>0$, then $p=1$ holds. Moreover, binding constraint $(\lambda)$ yields

$$
\Delta=\frac{R_{m^{G}+1}}{2(z-\underline{w})}(k+z-\underline{w}+\sqrt{k(k+z-\underline{w})})-k
$$

Constraint $(\alpha)$ requires $\Delta \geqslant z-\underline{w}$, that is $R_{m^{G}+1}(\sqrt{k+z-\underline{w}}+\sqrt{k}) \geqslant 2 \sqrt{k+z-\underline{w}}(z-\underline{w})$. However, this inequality does not hold because $R_{m^{G}+1}<\widehat{R}$ holds.

\section{References}

Auriol, E., \& Brilon, S. (2014). Anti-social behavior in profit and nonprofit organizations. Journal of Public Economics, 117, $149-161$.

Becker, G. S. (2009). A treatise on the family. Harvard University Press.

Besley, T., \& Ghatak, M. (2005). Competition and incentives with motivated agents. American Economic Review, 95, 617-636.

Bester, H., \& Hellwig, M. (1987). Moral hazard and equilibrium credit rationing: An overview of the issues, in Agency theory, information, and incentives (pp. 135-166). Berlin, Heidelberg: Springer.

Brookins, P., Goerg, S., \& Kube, S. (2017). Self-chosen goals, incentives, and effort (Unpublished manuscript).

Chakraborty, A., \& Citanna, A. (2005). Occupational choice, incentives and wealth distribution. Journal of Economic Theory, $122,206-224$.

Corgnet, B., Gómez-Miñambres, J., \& Hernán-Gonzalez, R. (2015). Goal setting and monetary incentives: When large stakes are not enough. Management Science, 61

(12), 2926-2944.

Dam, K., \& Pérez-Castrillo, D. (2006). The principal-agent market, Frontiers in Economic Theory. Berkeley Electronic Press, 2(1). Article 1.

Deci, E., \& Ryan, R. (1985). Intrinsic motivation and self-determination in human behavior. New York: Plenum.

Demange, G., \& Gale, D. (1985). The strategy structure of two-sided matching markets. Econometrica, 53, 873-888.

De Meza, D., \& Webb, D. C. (2007). Incentive design under loss aversion. Journal of the European Economic Association, 5(1), 66-92.

Dittmann, I., Maug, E., \& Spalt, O. (2010). Sticks or carrots? Optimal CEO compensation when managers are loss averse. Journal of Finance, 65(6), 2015-2050.

Flavin, M., \& Yamashita, T. (2002). Owner-occupied housing and the composition of the household portfolio. American Economic Review, 92(1), 345-362.

Gale, D., \& Shapley, L. S. (1962). College admissions and the stability of marriage. The American Mathematical Monthly, 69(1), 9-15.

Génicot, G., \& Ray, D. (2020). Aspirations and economic behavior. Annual Review of Economics, 12, 715-746.

Ghatak, M., Morelli, M., \& Sjostrom, T. (2001). Occupational choice and dynamic incentives. Review of Economic Studies, 68(4), 781-810.

Gilad, B., \& Levine, P. (1986). A behavioral model of entrepreneurial supply. Journal of Small Business Management, 24(4), 45-54.

Gómez-Miñambres, J. (2012). Motivation through goal setting. Journal of Economic Psychology, 33(6), 1223-1239.

Herweg, F., Muller, D., \& Weinschenk, P. (2010). Binary payment schemes: Moral hazard and loss aversion. American Economic Review, 100(5), 2451-2477.

Innes, R. D. (1990). Limited liability and incentives contracting with ex-ante action choices. Journal of Economic Theory, 52, $45-67$.

Kahneman, D., \& Tversky, A. (1979). Prospect theory: An analysis of decision under risk. Econometrica, 47, $263-292$.

Köszegi, B. (2014). Behavioral contract theory. Journal of Economic Literature, 52(4), 1075-1118.

Köszegi, B., \& Rabin, M. (2006). A model of reference-dependent preferences. The Quarterly Journal of Economics, 121(4), 1133-1165.

Kreps, D. M. (1997). Intrinsic motivation and extrinsic incentive. American Economic Review, 87(2), 1047-1073.

La Ferrara, E. (2019). Aspirations, social norms, and development. Journal of the European Economic Association, 17(6), 1687-1722.

Legros, P., \& Newman, A. F. (2007). Beauty is a beast, frog is a prince: Assortative matching with nontransferabilities. Econometrica, 75(4), 1073-1102.

Macho-Stadler, I., \& Pérez-Castrillo, D. (2018). Moral hazard: Base models and two extensions. In L. C. Corchón, \& M. A. Marini (Eds.), The Handbook of Game Theory and Industrial Organization (Vol. 1). Edward Elgar. https://link.springer.com/content/pdf/10.1007/s13209-020-00215-3.pdf.

Macho-Stadler, I., \& Pérez-Castrillo, D. (2020). Agency theory meets matching theory. SERIEs: Journal of the Spanish Economic Association (in press).

Poblete, J., \& Spulber, D. (2012). The form of incentive contracts: agency with moral hazard, risk neutrality, and limited liability. The RAND Journal of Economics, 43

(2), 215-234.

Quérou, N., Soubeyran, A., \& Soubeyran, R. (2020). Contracting under unverifiable monetary costs. Journal of Economics and Management Strategy, 29(4), 892-909.

Ray, D. (1998). Development economics. Princeton University Press.

Shapley, L. S., \& Shubik, M. (1971). The assignment game I: The core. International Journal of Game Theory, 1(1), 111-130.

Shetty, S. (1988). Limited liability, wealth differences and tenancy contracts in agrarian economies. Journal of Development Economics, $29(1), 1-22$.

Tirole, J. (2010). The theory of corporate finance. Princeton University Press.

Tversky, A., \& Kahneman, D. (1991). Loss aversion in riskless choice: A reference-dependent model. The Quarterly Journal of Economics, 106(4), 1039-1061.

Tversky, A., \& Kahneman, D. (1992). Advances in prospect theory: Cumulative representation of uncertainty. Journal of Risk and Uncertainty, 5(4), 297-323. 